Pacific Journal of Mathematics

SPACES WITH BASES SATISFYING CERTAIN ORDER AND 


\title{
SPACES WITH BASES SATISFYING CERTAIN ORDER AND INTERSECTION PROPERTIES
}

\author{
W. F. LiNDGREN AND P. J. NyIKOS
}

\begin{abstract}
An ortho-base is a base $\mathscr{B}$ such that the intersection of any subcollection is either an open set or a singleton for which the subcollection is a local base. This paper is primarily devoted to the relationship of bases of this sort to other topological properties of bases, and of the space itself. In $\$ 1$, we compare ortho-bases to bases having similar properties, and study the relationship with developability and paracompactness. In \$2, we define "rank" of a base for arbitrary cardinals and show how it is related to orthocompactness, ortho-bases, and bases of countable order. Section 3 treats two related ascending chain conditions in relation to bases of sub-infinite rank and ortho-bases. Section 4 relates the possession of an ortho-base to a number of "generalized metric" properties such as first countability, quasi-developability, and quasi-metrizability. The remaining two sections give examples illustrating the various properties and raise a number of unsolved problems.
\end{abstract}

1. Ortho-bases and related concepts. Throughout this paper "space" will always mean " $T_{1}$ topological space." Many of the spaces we will be studying have bases satisfying a rather strong property:

Definition 1.1. A base $\mathscr{B}$ for a space $X$ is an ortho-base if for each subcollection $\mathscr{A}$ of $\mathscr{B}$, either (i) $\cap \mathscr{A}$ is open or (ii) $\cap \mathscr{A}$ is a nonisolated singleton $\{x\}$ and $\mathscr{A}$ is a base for the neighborhoods of $x$.

It is easy to see that every point in a space with an ortho-base has a totally ordered open base for its neighborhoods. The proof of the following lemma is also easy and is omitted.

Lemma 1.2. Let $\mathscr{B}$ be an ortho-base for a space $X$.

(i) Every subset of $\mathscr{B}$ which is a base is an ortho-base.

(ii) The collection of all unions of chains in $\mathscr{B}$ is an ortho-base.

(iii) The collection of all open intersections of subsets of $\mathscr{B}$ is an ortho-base.

(iv) Given a subspace $Y$ of $X$, the collection of all sets of the form $Y \cap B$, with $B \in \mathscr{B}$ is an ortho-base for $Y$.

Concepts similar to that of an ortho-base have appeared in the recent literature. For example, Alexandroff introduced the concept of a 
uniform open base: a base $\mathscr{B}$ such that for every point $p$ and every neighborhood $U$ of $p$, there are most finitely many $V \in \mathscr{B}$ for which $p \in V, V-U \neq \varnothing[24]$. (Compare Lemma 1.3 below.) A space possesses a uniform base if, and only if, it is a metacompact developable space [1]. From this result and Bing's famous theorem on the metrizability of collectionwise normal developable spaces [3], there follows Alexandroff's well-known result that every collectionwise normal space with a uniform base is metrizable [1], [24].

A special kind of uniform open base is a regular open base [24]: a base $\mathscr{B}$ such that for every point $p$ and every neighborhood $U$ of $p$, there exists a neighborhood $W$ of $p$ such that there are at most finitely many $V \in \mathscr{B}$ for which $W \cap V \neq \varnothing$ and $V-U \neq \varnothing$. A space is metrizable if, and only if, it has a regular open base.

More general than "uniform open base" is the concept of a base of countable order [35]: each perfectly decreasing collection (i.e. every member of the collection has another member properly contained in it) of members of the base either has empty intersection or is a local base at some point. This concept and the preceding one are due to Arhangel'skii, who proved that a space is metrizable if, and only if, it is paracompact and has a base of countable order. Generalizing both this and the result on spaces with uniform bases, Wicke and Worrell showed [35] that a space is developable if, and only if, it is $\theta$-refinable and has a base of countable order.

Although every uniform base is an ortho-base, there are bases of countable order that are not ortho-bases. In fact, $\omega_{1}$ has a base of countable order, but no ortho-base (Example 5.4). On the other hand, $D_{1}^{*}$ (Example 5.3) has an ortho-base, but no base of countable order.

A concept which generalizes bases of countable order and is satisfied by all ortho-bases on spaces without isolated points was introduced by D. J. Lutzer in [21]: A base $\mathscr{B}$ for a space $X$ is a strong quasi-uniform base if every filterbase $\mathscr{F}$ of members of $\mathscr{B}$ satisfying $\cap F=\{p\}=$ $\bigcap\{\mathrm{cl} F \mid F \in \mathscr{F}\}$ is a neighborhood base at $p$. In the same paper, Lutzer introduced the slightly weaker and more complicated concept of quasi-uniform base and showed that a regular space is developable if, and only if, it is $\theta$-refinable and has a quasi-uniform base and a $G_{\delta}$ diagonal. One drawback of this concept is that the property of having a strong quasi-uniform base (unlike the earlier properties) is not hereditary [21].

The following easily proven lemma shows that an ortho-base can be regarded as a direct generalization of a uniform base.

LEMMA 1.3. A base $\mathscr{B}$ for a space $X$ is an ortho-base if, and only if, for each point $p$ of $X$ and each open set $U$ containing $x, \cap\{V \in \mathscr{B} \mid p \in$ $V, V-U \neq \varnothing\}$ is open. 
As a matter of notation, for each collection $\mathscr{C}$ of sets, let $\mathscr{C}_{p}$ denote $\{C \mid p \in C$ and $C \in \mathscr{C}\}$. Let $\eta(p)$ denote the set of all neighborhoods of $p$.

Definition 1.4. A cover $\mathscr{C}$ of a space $X$ is a $Q$-cover if, and only if, for each $x$ in $X, \cap \mathscr{C}_{x}$ is open. A space $X$ is said to be orthocompact if for each open cover $\mathscr{C}$ of $X$ there is a $Q$-cover $\mathscr{R}$ which refines $\mathscr{C}$.

The choice of the name ortho-base was made in part because of the ease with which it can be shown that every space with an ortho-base is orthocompact (Lemma 2.5).

One important class of spaces with ortho-bases is the class of orthocompact developable spaces. In fact:

THEOREM 1.5. Let $X$ be developable. The following are equivalent:

(i) $X$ is orthocompact.

(ii) $X$ has an ortho-base.

(iii) $X$ is hereditarily orthocompact.

Proof. (i) $\Rightarrow$ (ii) Let $\mathscr{G}_{n}$ be a development for $X$ such that for each $n$ in $N$ and each $x$ in $X, \operatorname{St}\left(x, \mathscr{G}_{n+1}\right) \subset \operatorname{St}\left(x, \mathscr{G}_{n}\right)$. For each $n$ in $N$, let $\mathscr{H}_{n}$ be a $Q$-refinement of $\mathscr{G}_{n}$. We will show that $B=\cup_{n=1}^{\infty} \mathscr{H}_{n}$ is an ortho-base for $X$. Let $\mathscr{A} \subset \mathscr{B}$ and suppose $p \in \cap \mathscr{A}$. There are two cases: (i) $\mathscr{A} \cap \mathscr{H}_{n} \neq \varnothing$ for only finitely many $n$ 's. It is evident in this instance that $\cap \mathscr{A}$ is open. (ii) $\mathscr{A} \cap \mathscr{H}_{n} \neq \varnothing$ for infinitely many $n$ 's. Let $G \in \eta(p)$ be arbitrary; for some $n \in N$, St $\left(p, \mathscr{G}_{n}\right) \subset G$. For some $m>n, \mathscr{A} \cap \mathscr{H}_{m} \neq \varnothing$; then

$$
p \in R \subset \operatorname{St}\left(p, \mathscr{G}_{m}\right) \subset \operatorname{St}\left(p, \mathscr{G}_{n}\right) \subset \mathscr{G}
$$

This establishes that $\mathscr{A}$ is a base for $\eta(p)$.

(ii) $\Rightarrow$ (iii) This will follow from Lemma 2.5 .

Additional conditions equivalent to (i), (ii), and (iii) may be found in [9, p. 7621.

Definition 1.6. Let $(X, \mathscr{T})$ be a topological space and let $M$ be a subset of $X$. The discretization of $X$ by $M$ is the space whose open sets are of the form $U \cup B$ where $U \in \mathscr{T}$ and $B \subset M$.

The discretization of $X$ by $M$ is also referred to sometimes as "the space obtained from X by scattering the points of $M$ ". It is denoted by $X_{M}$ in [8], where some of the properties of discretizations, including conditions for the preservation of normality, may be found. Here we 
are primarily interested in the fact that every discretization of a space with an ortho-base likewise has an ortho-base. In fact, the addition of the set of isolated singletons to an ortho-base gives an orthobase. Thus, for example, the Michael line, the discretization of the real line obtained by isolating each irrational number, has an orthobase. Furthermore if $X$ has an ortho-base, and if $Y$ is a space obtained by replacing isolated points of $X$ by clopen subsets, each with an ortho-base in the relative topology, then $Y$ also has an ortho-base. This process can be iterated transfinitely, with inverse limits taken at limit ordinals, and what is obtained at each step is a space with an ortho-base.

If in addition, the spaces involved are all paracompact, then the spaces obtained at any step are also paracompact. However, a much stronger result obtains in this case: every paracompact space with an ortho-base can be obtained by a process of the sort just indicated, where $X$ and all the spaces which replace isolated points are metrizable. This is one reason for the choice of words in the following definition.

Definition 1.7. A proto-metrizable space is a paracompact space with an ortho-base.

Proto-metrizable spaces have a very rich structure theory, which will be expounded upon in a paper now in preparation [28]. Most of the results in that paper may be found (without proof) in [25].

One subclass of proto-metrizable spaces, the non-Archimedean spaces, has been the subject of several articles already [2], [6], [26]. One characterization of these spaces is that they are the ultraparacompact spaces with ortho-bases. (A space $X$ is ultraparacompact if every open cover of $X$ can be refined to a partition of $X$ into clopen subsets.) Another is that they are precisely the spaces obtainable from ultrametrizable spaces (ultraparacompact metric spaces) in the same way as proto-metrizable spaces may be obtained from all metric spaces. The usual definition, however, states that they are the spaces with bases of rank one. (Definition 2.2, below)

For the remainder of this paper, we will be concerned with those properties of spaces with ortho-bases that can be obtained without assuming paracompactness.

2. Ranks of systems of sets. In this section, we extend the concept of "rank" as defined by Nagata [23] and prove some simple results relating it to the concepts treated in $\$ 1$.

Definition 2.1. A collection $\mathscr{A}$ of subsets of a set $X$ is incomparable if, given any two members $A_{1}$ and $A_{2}$ of $\mathscr{A}$ neither $A_{1} \subset A_{2}$ nor $A_{2} \subset A_{1}$ obtains. 
Definition 2.2. Let $m$ be a cardinal number. A collection $\mathscr{A}$ of subsets of a set $X$ is of rank $\leqq m$ at $x$ in $X$ if every incomparable subcollection $\mathscr{A}^{\prime}$ of $\mathscr{A}$, each member of which contains $x$, is of cardinal $\leqq \mathrm{m}$. The collection $\mathscr{A}$ is of rank $\mathrm{m}$ at $x$ if it is of rank $\leqq \mathrm{m}$ at $x$ and if there exists an incomparable subcollection $\mathscr{A}^{\prime}$ of $\mathscr{A}$ of cardinal $\mathrm{m}$, such that each member of $\mathscr{A}^{\prime}$ contains $x$.

If $m$ is a limit cardinal, $\mathscr{A}$ is of rank $(m-)$ at $x$ if it is of rank $\leqq m$ at $x$, is not of rank $\mathrm{n}$ at $x$ for each $\mathrm{n}<\mathrm{m}$, and there exists a set $\mathscr{A}^{\prime}$ of incomparable elements of $\mathscr{A}$, each of which contains $x$, with $|\mathscr{A}|>n$.

In other words, $\mathscr{A}$ is of rank $\mathrm{m}$ at $x$ if $\mathrm{m}$ is the maximum number of incomparable members of $\mathscr{A}$ containing $x$. It is of rank $(m-)$ if $m$ is the unattained supremum of the number of incomparable members of $\mathscr{A}$ containing $x$.

Definition 2.3. Let $\mathrm{m}$ be a cardinal number, $X$ a set, $\mathscr{A}$ a collection of subsets of $X$. The collection of $\mathscr{A}$ is of rank $\leqq m$ if $\mathscr{A}$ is of rank $\leqq m$ at every point of $X$. It is of rank $\mathrm{m}$ if $\mathscr{A}$ is of rank $\leqq \mathrm{m}$ and $\mathscr{A}$ is of rank $m$ at some point $X$. If $m$ is a limit cardinal, then $\mathscr{A}$ is of rank $(m-)$ if $\mathscr{A}$ is of rank $\leqq m$, is not of rank $m$, and is of rank $(m-)$ at some point of $X$; and $\mathscr{A}$ is of rank ( $m--)$ if $\mathscr{A}$ is of rank $\leqq m$, is not of rank $\mathrm{m}$ nor of rank $(\mathrm{m}-)$, and for every $\mathrm{n}<\mathrm{m}$ there is a point of $X$ at which $\mathscr{A}$ is of rank $\geqq n$.

A collection of some finite rank $n$ or of rank $\left(\boldsymbol{N}_{0}-\right)$ or of rank $\left.\left(\boldsymbol{N}_{0}-\right)^{-}\right)$will be said to be of sub-infinite rank.

The spaces with rank one bases are the non-Archimedean spaces. (In fact, this is the usual definition of a non-Archimedean space.) As mentioned above, they are the ultraparacompact spaces with ortho-bases - in particular, every rank one base is an ortho-base. [2], [26]. This result does not even extend to bases of rank 2, although some of the properties of ortho-bases still hold; for example:

LEMMA 2.4. If $X$ be a space with a base $\mathscr{B}$ of finite rank, then every point of $X$ has a totally ordered local base.

Proof. Let $\mathscr{B}$ be of rank $n$ at $p$. Let $\mathscr{C}_{1}$ be a maximal chain of members of $\mathscr{B}_{p}$. If $\mathscr{C}_{1}$ is not a local base at $p$, there exists a neighborhood $N_{1}$ of $p$ containing no member of $\mathscr{C}_{1}$. Let $\mathscr{B}_{p}^{1}$ be the set of all members of $\mathscr{B}_{p}$ that are contained in $N_{1}$. Then $\mathscr{B}_{p}^{1}$ is of rank $\leqq n-1$ (at p). (Indeed, suppose $B_{1}, B_{2}, \cdots, B_{k}$ are incomparable members of $\mathscr{B}_{p^{\text {. }}}^{1} \quad$ By maximality of $\mathscr{C}_{1}$, there is, for each $i$, a member $\mathscr{C}_{i}$ of $\mathscr{C}_{1}$ such that $B_{\imath}-C_{\imath} \neq \varnothing$. The least of these $C_{\imath}$ 's is incomparable with all $B_{i}$, 
$1 \leqq i \leqq k$.) $\quad$ Let $\mathscr{C}_{2}$ be a maximal chain in $\mathscr{B}_{p}^{1}$. Continue thus for all $k$, letting $C_{k}$ be a maximal chain of members of $\mathscr{B}_{p}^{k-1}, N_{k}$ a neighborhood of $p$ containing no member of $\mathscr{C}_{k}$, and $\mathscr{B}_{p}^{k}$ the set of all members of $\mathscr{B}_{p}$ contained in $N_{k}$. The process comes to an end in at most $n$ steps, with a chain $\mathscr{C}_{k}$ that is a local base at $p$.

It is actually possible to prove that $\mathscr{B}_{p}$ is a union of at most $n$ chains, but this is a nontrivial result [7]. Lemma 2.4 clearly extends to bases of rank $\boldsymbol{N}_{0}--$, but not to all bases of sub-infinite rank. See Example 5.3.

The following lemma has been deferred from $\$ 1$ to pave the way for Lemma 2.6.

\section{LEMMA 2.5. Every space with an ortho-base is orthocompact.}

Proof. Let $\mathscr{A}$ be an open cover of $X$, whose members we may assume belong to the ortho-base. By a straightforward use of wellordering and transfinite induction, we may obtain a subcover $\mathscr{B}$ of $\mathscr{A}$ such that each $B$ in $\mathscr{B}$ contains a point belonging to no predecessor of $B$. Since any descending sequence of members of $\mathscr{B}$ is finite, $\mathscr{B}$ obviously contains no local base at any nonisolated point of $X$. Therefore, $\mathscr{B}$ is a $Q$-cover.

LEMMA 2.6. Suppose $\mathscr{A}$ is an open cover of $X$ such that for each $p$ in $X$, either (i) $\cap \mathscr{A}_{p} \in \eta(p)$ or (ii) $\mathscr{A}_{p}$ is of sub-infinite rank. Then $\mathscr{A}$ has a $Q$-subcover.

Proof. Let $\mathscr{A}$ be well-ordered and satisfy (i) or (ii) at each point. Define $\mathscr{B}$ with respect to $\mathscr{A}$ as in the proof of Lemma 2.5. Suppose $\mathscr{B}$ is not a $Q$-cover; then for some subcollection $\mathscr{C}$ of $\mathscr{B}$, $\cap \mathscr{C}$ is not open. Say $p \in \cap \mathscr{C}$ and $\cap \mathscr{C}$ is not a neighborhood of p. Let $C_{1}$ denote the first element of $\mathscr{C}$. Set $\mathscr{C}_{2}=\{C \mid C \in \mathscr{C}$ and $\left.C_{1} \not \subset C\right\}$; then $\cap \mathscr{C}=C_{1} \cap \cap \mathscr{C}_{2}$, hence $p \in \cap \mathscr{C}_{2} \notin \eta(p)$. Let $C_{2}$ denote the first element of $\mathscr{C}_{2}$. Inductively, let $C_{n}$ denote the first element of $\mathscr{C}_{n}$ and set $\mathscr{C}_{n+1}=\left\{C \mid C \in \mathscr{C}_{n}\right.$ and $\left.C_{n} \not \subset C\right\}$. Then $\cap \mathscr{C}_{n}=$ $C_{n} \cap \cap \mathscr{C}_{n+1}$; hence $p \in \cap \mathscr{C}_{n+1} \notin \eta(p)$. In this way, we obtain a sequence $\left(C_{n}\right)$ in $\mathscr{C}$ such that $p \in \bigcap_{n=1}^{\infty} C_{n}$ and $\left\{C_{n} \mid n \in \mathbf{N}\right\}$ is incomparable. This is a contradiction since $\left\{C_{n} \mid n \in \mathbf{N}\right\}$ is a subcollection of $\mathscr{A}_{p}$ that has sub-infinite rank.

COROllary 2.7. A space is orthocompact if, and only if, every open cover has a refinement satisfying (i) or (ii) at each point.

COROLlary 2.8. Every space with a base of sub-infinite rank is orthocompact. 
Problem. Is every space with a base of sub-infinite rank metacompact?

It can be shown [14] that every space with a base of finite rank is (hereditarily) metacompact. Metacompactness also obtains, and much more easily, in spaces with bases that, in addition to being of sub-infinite rank, also satisfy the ascending chain condition. This is one result of the following section.

THEOREM 2.9. If $X$ has a base of countable order and a base of sub-infinite rank, then $X$ has an ortho-base.

Proof. By Theorem 3.4 of [36], there is a base $\mathscr{B}$ that is simultaneously a base of countable order and a base of sub-infinite rank. Suppose there exists $\mathscr{A} \subset \mathscr{B}$ such that $\cap \mathscr{A}$ is not open for some $p \in \cap \mathscr{A}, \mathscr{A}$ is not a base at $p$. Then $\mathscr{A}$ is not perfectly decreasing; hence there exists $A_{1} \in \mathscr{A}$ such that $A \not \subset A_{1}$ for all $A$ in $\mathscr{A}$. Set $\mathscr{A}_{1}=\{A \mid A \in \mathscr{A}$ and $\left.A_{1} \not \subset A\right\}$. Since $\cap \mathscr{A}=A_{1} \cap \cap \mathscr{A}_{1}$ and $\mathscr{A}_{1} \subset \mathscr{A}, \mathscr{A}_{1}$ is not a base at $p$ and hence $\mathscr{A}_{1}$ is not perfectly decreasing. Inductively choose $A_{n+1} \in \mathscr{A}_{n}$ such that $A \not \subset A_{n+1}$ for all $A$ in $\mathscr{A}_{n}$. Set $\mathscr{A}_{n+1}=\left\{A \mid A \in \mathscr{A}_{n}\right.$ and $\left.A_{n+1} \not \subset A\right\} . \cap \mathscr{A}_{n+1}=A_{n} \cap \cap \mathscr{A}_{n}$ and $\mathscr{A}_{n+1} \subset \mathscr{A}_{n}$ hence $\mathscr{A}_{n+1}$ is not a base at $p$ and $\mathscr{A}_{n+1}$ is not perfectly decreasing. In this way, we obtain a sequence $\left(A_{n}\right)$ in $\mathscr{A}$ such that no two elements of $\left(A_{n}\right)$ are comparable and $p \in \bigcap_{n=1}^{\infty} A_{n}$. This contradicts the assumption that $\mathscr{B}$ has subinfinite rank.

It may be possible to strengthen this result considerably: if it could be shown that every space with a base of sub-infinite rank is metacompact [or even $\theta$-refinable: see Problem 6.3], then we could show that a space is metacompact and developable if, and only if, it has a base of countable order and a base of sub-infinite rank, in a manner similar to the proof of the following theorem.

THEOREM 2.10. If $X$ has a base of countable order and a base of finite rank, then $X$ is metacompact and developable.

Proof. Every space with a base of finite rank is metacompact [14]. By the result of Wicke and Worrell, if $X$ then has a base of countable order, it is developable.

\section{Ascending chain conditions.}

Definition 3.1. Let $\mathscr{A}$ be a collection of subsets of a set $X$. $\mathscr{A}$ is Noetherian if every ascending sequence $A_{1} \subset A_{2} \subset \cdots$ of members of $\mathscr{A}$ 
is finite. $\mathscr{A}$ is upper-chain-complete if whenever $\mathscr{C}$ is a chain in $\mathscr{A}$, then $\cup \mathscr{C} \in \mathscr{A}$.

The terms lower-chain-complete and chain-complete are defined analogously, but we will have no occasion to use these latter two concepts in this paper. Arhangel'skii has used "Noetherian" to refer to collections that are upper-chain-complete. However, because of the wide usage of "Noetherian" (in our sense of the word) in ring theory and lattice theory, it is best that the usage of 3.1 be adopted. It is trivial to show (as in ring theory) that a collection is Noetherian if, and only if, every subcollection has a maximal element.

Every product of spaces with Noetherian bases is easily seen to possess such a base; and if $X$ has a Noetherian base, every open subspace obviously has one. On the other hand, not every closed subspace of a space with a Noetherian base has a Noetherian base. For example, the spaces of Example 5.5 can be embedded as closed subspaces of an open subspace of a product of two-point spaces.

Lemma 3.2. Let $\mathrm{m}$ be a countable cardinal, and let $\mathscr{A}$ be a collection of subsets of a set $X$. The following are equivalent: (i) $\mathscr{A}$ is Noetherian, of rank $<m$ at $x$, (ii) Given a set $\mathscr{A}^{\prime}$ of members of $\mathscr{A}$ each containing $x$, there exists a subset $\mathscr{A}^{\prime \prime}$ of $\mathscr{A}^{\prime}$ such that every other member of $\mathscr{A}^{\prime}$ is contained in some member of $\mathscr{A}^{\prime \prime}$, and $\left|\mathscr{A}^{\prime \prime}\right|<\mathrm{m}$.

Proof. To show that the first condition implies the second, let $\mathscr{A}^{\prime}$ be a subset of $\mathscr{A}$, each containing a fixed point $x$. Let $\mathscr{A}^{\prime \prime}$ be the set of all maximal members of $\mathscr{A}^{\prime}$ containing $x$. Since no two members of $\mathscr{A}^{\prime \prime}$ are comparable, the cardinality of $\mathscr{A}^{\prime \prime}$ is $<\mathrm{m}$.

Conversely, if $\mathscr{A}$ satisfies (ii) it is clearly of rank $<\mathfrak{m}$, and no strictly ascending chain of members of $\mathscr{A}$ can be infinite.

THEOREM 3.3. Every space with a Noetherian base of sub-infinite rank is (hereditarily) metacompact.

Proof. Let $\mathscr{B}$ be a Noetherian base of sub-infinite rank for the topology on $X$. Let $U$ be an open cover of $X$, and for each point $x$ let $\mathscr{B}(x)$ be the (finite) collection of maximal members of $\mathscr{B}$ that contain $x$ and are contained in some member of $\mathcal{u}$. Let $\mathscr{V}=$ $\cup\{\mathscr{B}(x) \mid x \in X\}$. This is a point-finite cover, since the members of $\mathscr{V}$ that contain $x$ are the sets in $\mathscr{B}(x)$.

EXAMPLE 3.4. Every uniform base is Noetherian, of sub-infinite 
rank. Hence a Moore space is (hereditarily) metacompact if, and only if, it has a Noetherian base of sub-infinite rank.

Theorem 3.3 is especially significant because:

THEOREM 3.5. The finite product of spaces, each of which has a Noetherian base of sub-infinite rank, likewise has a Noetherian base of sub-infinite rank.

A proof may be had by dualizing Exercise 7, p. 181 of [4] and applying it to members of a base containing a fixed point. Details are spelled out in [14]. If one omits the word "Noetherian" from Theorem 3.5 , it becomes false.

EXAMPLE 3.6. The Sorgenfrey line has a Noetherian base and a base of sub-infinite rank, but not a base satisfying both simultaneously, because the Sorgenfrey plane does not have a base of sub-infinite rank at all. In fact, every base for the Sorgenfrey plane is of rank $\geqq c$. See Example 5.2.

Here are four more theorems tying together some of the concepts we have introduced. The first two have easy proofs by transfinite induction. Proofs of the other two will appear in a forthcoming paper.

THEOREM 3.8. Let $X$ be a space with a base of finite rank $n$. Then $X$ has a rank $n$ base that is upper-chain-complete.

THEOREM 3.9. Let $X$ be a space with an ortho-base. Then $X$ has an ortho-base that is upper-chain-complete.

THEOREM 3.10. Let $X$ be a space with an ortho-base $\mathscr{B}$ and a base $V$ of (finite) rank $n$. Then $X$ has a Noetherian base of rank $n$.

THEOREM 3.11. Let $X$ be a space with an ortho-base of (finite) rank $n$. Then $X$ has a Noetherian ortho-base of rank $n$.

4. Condensation theorems. A space $Y$ can be condensed onto a space $X$ if it admits a one to one continuous function onto $X$ (in other words, $X$ admits a finer topology making it homeomorphic to $Y)$. Some properties of a space can of course be preserved under the taking of a finer topology, such as the Hausdorff separation axiom and total disconnectedness.

If $Y$ has an ortho-base, several properties of $X$ are preserved that are not always preserved otherwise. For example, if $X$ is first counta- 
ble, then every point of $Y$ is a $G_{\delta}$, and it follows from the existence of an ortho-base that $Y$ is first countable. In fact:

THEOREM 4.1. [25] Let $X$ be a space with an ortho-base. The following are equivalent:

(i) $X$ is first countable.

(ii) $X$ is sequential.

(iii) Every point of $X$ is $a G_{\delta}$.

(iv) $X$ is a $k$-space.

(v) $X$ is a $q$-space.

A way of proving this theorem is indicated in Example 5.3.

In this section, we will treat similar phenomena.

Let $\left(\mathscr{G}_{n}\right)$ be a sequence of collections of open subsets of space $X$, indexed by the positive integers. Consider the following properties this sequence might have:

(I) $\quad \mathscr{G}_{n}$ is a cover of $X$ for each $n$.

(II) For each point $p$ of $X$ the set $\operatorname{St}\left(p, \mathscr{G}_{n}\right)$ [by $\operatorname{St}\left(p, \mathscr{G}_{n}\right)$ is meant the union of all sets in $\mathscr{G}_{n}$ which contain $p$ ] is nonempty for infinitely many $n$.

(III) For each pair $p, q$ of points of $X$, there exists $n$ such that $\operatorname{St}\left(p, \mathscr{G}_{n}\right) \neq \varnothing$ and $q \notin \operatorname{St}\left(p, \mathscr{G}_{n}\right)$.

(IV) The nonempty sets of the form $\operatorname{St}\left(p, \mathscr{G}_{n}\right)$ form a local base at $p$ for all $p$ in $X$.

(V) If a point $x_{n}$ is chosen from each nonempty set of the form $\operatorname{St}\left(p, \mathscr{G}_{n}\right)$, then $p$ is a cluster point of $\left(x_{n}\right)$.

For all spaces, (V) is equivalent to (IV) together with (II), and (IV) implies (III). The existence of a sequence satisfying (III) gives the definition of a quasi- $G_{\delta}$-diagonal [18], while existence of a sequence satisfying (I) and (III) together is easily shown to be equivalent to the possession of a $G_{\delta}$-diagonal (that is, the diagonal is a countable intersection of open sets in $X \times X)$. Possession of a sequence satisfying (I) and (IV), or one satisfying (I) and (V), characterizes the developable spaces, while condition (IV) or (V) alone gives the definition of a quasidevelopable space.

THEOREM 4.4. Let $X$ be a space with an ortho-base. The following are equivalent.

(i) $X$ is quasi-developable.

(ii) $X$ can be condensed onto a quasi-developable space.

(iii) $X$ has a quasi-G-diagonal.

Proof. That (i) implies (ii) and (ii) implies (iii) are true for arbitrary spaces. Now if $X$ has an ortho-base $\mathscr{B}$ and a sequence $\mathscr{G}_{n}$ satisfying 
(III), we replace it by a sequence of collections $\mathscr{G}_{n}^{\prime}$ such that for each $n$, $\bigcup \mathscr{G}_{n}=\bigcup \mathscr{G}_{2 n}^{\prime}$, such that each member of $\mathscr{G}_{2 n}^{\prime}$ is contained in some member of $\mathscr{G}_{n}$, such that $\mathscr{G}_{2 n+1}$ (for any $n$ ) is the collection of all isolated points of $X$, and such that $\mathscr{G}_{n}^{\prime}$ consists only of members of $\mathscr{B}$ (for all $n$ ).

If $p$ is an isolated point, $(\mathrm{V})$ is clearly satisfied at $p$. Otherwise, $x_{n}$ is chosen from each nonempty set of the form $\operatorname{St}\left(p, \mathscr{G}_{n}^{\prime}\right)$. Let $G_{n}$ be a member of $\mathscr{G}_{n}^{\prime}$ containing $x_{n}$. Then the $G_{n}$ 's, which intersect in $\{p\}$ by (III), form a local base at $p$, and $x_{n}$ clusters at $p$.

As the Michael line shows, we cannot obtain the analogous theorem for $G_{\delta}$-diagonals because of the special role of isolated points. However, what we can obtain may be even more interesting:

THEOREM 4.3. The following conditions are equivalent on a Hausdorff space $X$ that has an ortho-base.

(i) $X$ is a developable space.

(ii) $X$ is a semi-metrizable space.

(iii) $X$ is a semi-stratifiable space.

(iv) $X$ has a $G_{\delta}$-diagonal and its isolated points form an $F_{\sigma}$-set.

Proof. The implications (i) $\Rightarrow$ (ii) $\Rightarrow$ (iii) $\Rightarrow$ (iv) are well known and do not require the existence of an ortho-base. (Only the last requires the Hausdorff assumption.)

Finally, to show (iv) implies (i): we replace the sequence $\mathscr{G}_{n}$ with $\mathscr{G}_{n}^{\prime}$ as in the proof of Theorem 4.2, but this time we let $\mathscr{G}_{2 n}^{\prime}$ be a collection of isolated points whose union is closed, together with the complement of this union, arranging it so that $\bigcup_{n=1}^{\infty} \mathscr{G}_{2 n}^{\prime}$ includes all isolated points of $X$. The resulting collection of covers satisfies (I) and (V).

In the case where the developable space is metrizable, there is a theorem in the folklore which, among other things, shows how the Michael line fits into the general scheme of normality of products.

THEOREM 4.4. Let $X$ be a metrizable space and let $X_{M}$ be a discretization of $X$. The following are equivalent:

(v) $X_{M}$ is metrizable.

(vi) $\quad M$ is an $F_{\sigma}$-subset of $X_{M}$.

(vii) $M$ is an $F_{\sigma}$-subset of $X$.

(viii) The product of $X_{M}$ with any metrizable space is normal.

Moreover, all these are equivalent (with $X_{M}$ playing the role of $X$ ) to (i) through (iv) of Theorem 4.2. 
Proof. (v) is equivalent to (vi) by the Bing-Nagata-Smirnov metrization theorem: the addition of $\{\{x\} \mid x \in M\}$ to a $\sigma$-discrete base for $X$ yields a metrizable space if, and only if, $M$ is $\sigma$-discrete.

(vi) is equivalent to (i), (ii), (iii) and (iv). Let $\mathscr{B}$ be an ortho-base for $X$, then $\mathscr{B} \cup\{\{x\} \mid x \in M\}$ is an ortho-base for $X_{M}$, and $X_{M}$ has a $G_{\delta}$-diagonal. Hence (vi) is equivalent to (iv) and thus the other three conditions.

(vi) is equivalent to (vii). If $A$ is a closed subset of $X_{M}$, then its closure in $X$ does not contain any points outside $A \cup M$. Now the implication (vi) $\Rightarrow$ (vii) is immediate, while the other implication is trivial.

(v) $\Rightarrow$ (viii) This implication is trivial.

(viii) $\Rightarrow$ (vi) Suppose that $M$ is not an $F_{\sigma}$-set. Take $M$ in the relative topology of $X$ and look at its product with $X_{M}$. Let $A=$ $\{(m, m) \mid m \in M\}$ and $B=\left(X_{M}-M\right) \times M . \quad A$ and $B$ are disjoint closed sets. The proof given in [22] can be adapted to prove that $X_{M} \times M$ is not normal by showing that any open set containing $A$ has some point of $B$ in its closure. In fact, E. Michael points this out in a footnote.

A classification scheme, similar to (I) through (IV) above, which unifies certain generalizations of metric spaces, was first devised by R. W. Heath in [15] to characterize semi-metric and developable spaces. Simply put, the scheme is this: Let $(X, \mathscr{T})$ be a topological space and $g: N \times X \rightarrow \mathscr{T}$ be a function such that for all natural numbers $n$ and all points $x$ in $X, x \in g(n, x)$. It is often the case that $X$ has some property (e.g. developability) if, and only if, there exists such a function $g$ with some easily stated property. This idea has been exploited further by Hodel [16], [17] who not only has characterized several known topological properties in terms of such functions, but further has used such functions to define important new classes of spaces. (We have particularly in mind here the so-called $\beta$-spaces.)

Some properties $g$ could have are of special relevance to this section; for example:

(A) $\{g(n, x) \mid n \in \mathbf{N}\}$ is a local base at $x$.

(A') If $x_{n} \in g(n, x)$ then $x$ is a cluster point of the sequence $\left\langle x_{n}\right\rangle$.

(B) $\bigcap_{n=1}^{\infty} g(n, x)=\{x\}$.

(C) If $y \in g(n, x)$, then $g(n, y) \subset g(n, x)$.

(D) If $y \in g(n+1, x)$, then $g(n+1, y) \subset g(n, x)$.

(E) For each $x$ and each $n$, there exists $m$ such that if $y \in g(m, x)$, the set $g(m, y)$ is contained in $g(n, x)$.

Clearly, (A) and ( $\left.\mathrm{A}^{\prime}\right)$ are equivalent, and possession of such a sequence characterizes first countable spaces. Existence of a function $g$ satisfying (A) and (C) characterizes those spaces that admit a non- 
Archimedean quasi-metric, [11]. (Such spaces are called strongly first countable in [16].) Spaces that admit a separating $\sigma$ - $Q$-cover (called $\sigma^{*}$-spaces in [31] and $\alpha$-spaces in [17]) are characterized by the existence of a function $g$ satisfying (B) and (C). (Recall that a cover $\mathcal{U}$ of $X$ is separating if given two points $x$ and $y$ in $X$, there exists $U$ in $U$ such that $x \in U$ and $y \notin U$.) It is easily seen that a space admits a separating $\sigma$ $Q$-cover if, and only if, it can be condensed onto a space that admits a non-Archimedean quasi-metric. H. Ribeiro [29] has shown that a space is quasi-metrizable if and only if, it admits a function satisfying (A) and (D); while $\gamma$-spaces [16], [10] can be characterized by the existence of a function satisfying (A) and (E). Kofner [20] has exhibited a quasi-metric space that does not admit a non-Archimedean quasi-metric. It is not known whether every $\gamma$-space is quasi-metrizable.

To study the preservation of these properties under condensation, we have a lemma that allows us to substitute members of an ortho-base for open sets while preserving some important containments.

Lemma 4.5. Let $X$ be a space with an ortho-base. For each point $x$ of $X$, and each open neighborhood $U$ of $x$, it is possible to associate an open neighborhood $G(x, U)$ of $x$ in such a way that the sets $G(x, U)$ form an ortho-base for $X$ and

(i) $x \in G(x, U) \subset U$.

(ii) If $V \subset U$ and $x \in V$, then $G(x, V) \subset G(x, U)$.

(iii) If $y \in G(x, U)$, then $G(y, U) \subset G(x, U)$.

Proof. Let $\mathscr{B}$ be an ortho-base on $X$, closed under unions of chains. For each open set $U$ and each point $x$ of $U$, let $G(x, U)$ be the intersection of all members of $\mathscr{B}$ which contain $x$, are contained in $U$, and are maximal with respect to these two properties. Now (i) is clearly satisfied, and the set $G(x, U)$ is open because it is the intersection of incomparable members of $\mathscr{B}$. The set of all $G(x, U)$ is an ortho-base because it is a subset of an ortho-base and is a base because of (i). Condition (ii) is satisfied because each maximal member of $\mathscr{B}$ containing $x$ and contained in $V$ is contained in a maximal member of $\mathscr{B}$ containing $x$ and contained in $U$. Finally, (iii) is satisfied because every maximal member of $\mathscr{B}$ containing $x$ and contained in $U$ contains $y$ - the intersection $G(y, U)$ is taken over a bigger collection.

One might rephrase the following theorem by saying that if a space $X$ has an ortho-base and a function $g$ satisfying (B) and any of (C), (D), or (E), then it admits a function satisfying (A) together with (C), or (D), or $(\mathrm{E})$, respectively.

THEOREM 4.6. Let $X$ be a space with an ortho-base.

(i) If $X$ is a $\sigma^{*}$-space, it admits a non-Archimedean quasi-metric. 
(ii) If $X$ can be condensed onto a quasi-metric space, it is quasimetrizable.

(iii) If $X$ can be condensed onto a $\gamma$-space, it is a $\gamma$-space.

Proof. Let $G$ be as in Lemma 4.5 and let $g^{\prime}(n, x)=\{x\}$ if $x$ is isolated, and $g^{\prime}(n, x)=G(x, g(n, x))$ otherwise. By Lemma 4.5 (i), the function $g^{\prime}$ satisfies (A) whenever $g$ satisfies (B).

(i) Suppose $g$ satisfies (C). If $y \in g^{\prime}(n, x)$, then $y \in g(n, x)$ and $g(n, y) \subset g(n, x)$. Hence, by Lemma 4.5,

$$
g^{\prime}(n, y)=G(y, g(n, y)) \subset G(y, g(n, x)) \subset G(x, g(n, x))=g^{\prime}(n, x) .
$$

Hence $g^{\prime}$ satisfies $(C)$.

(ii) Clearly, $X$ can be condensed onto a quasi-metric space if, and only if, it admits a function $g: \mathbf{N} \times X \rightarrow \mathscr{T}$ satisfying (B) and (D). So if $y \in g^{\prime}(n+1, x)$, then (Lemma 4.5)

$$
\begin{array}{r}
g^{\prime}(n+1, y)=G(y, g(n+1, y)) \subset G(y, g(n, x)) \\
\subset G(x, g(n, x))=g^{\prime}(n, x) .
\end{array}
$$

Thus $g^{\prime}$ satisfies (D).

The proof of (iii) is similar.

5. Examples. In this section we discuss some well-known spaces from the point of view of the theory that has been presented here. They include a space with a rank 1 base and a $G_{\delta}$-diagonal that is not a Moore space; a space with a base of sub-infinite rank and a Noetherian base, but no Noetherian base of sub-infinite rank; a space with a Noetherian base of sub-infinite rank but no base of finite rank; a space with a Noetherian base but no base of sub-infinite rank; and a space with no Noetherian base.

EXAMPLE 5.1. The Michael line was introduced [22] as an example of a paracompact space whose product with a metric space (the space of irrational numbers) is not normal. It is the discretization $\mathbf{R}_{M}$ where $M$ is the set of irrational numbers. (Definition 1.6) A rank 1 base $\mathscr{V}$ (hence an ortho-base) for Michael line, easily seen to be Noetherian, is obtained by picking an irrational number $y$, and for each positive integer $n$ letting

$$
\mathscr{V}_{n}=\{] y+k / 2^{n}, y+(k+1) / 2^{n}[\mid k \in \mathbf{Z}\}
$$

(] $a, b$ [ denotes the open interval from $a$ to $b$.) letting $\mathscr{V}_{0}$ be the set of all irrational singletons and $\mathscr{V}=\bigcup_{n=0}^{\infty} \mathscr{V}_{n}$. 
If we define $\mathcal{U}_{n}$ analogously to $\mathscr{V}_{n}$ but with 0 substituted for $y$, then we obtain a Noetherian rank 1 base for the space of irrational numbers.

By Theorem 3.5, the product of the Michael line with the space of irrationals and the product of the Michael line with itself both have Noetherian bases of sub-infinite rank and are therefore metacompact (even though the former is not paracompact). However, a direct argument. establishes even more. Let

$$
\mathscr{W}_{n}=\left\{U \times V \mid U \in \mathscr{U}_{n} \text { and } V \in \mathscr{V}_{n}\right\}
$$

and let $\mathscr{Z}_{n}=\left\{U \times V \mid U \in \mathscr{U}_{n}\right.$ and $\left.V \in \mathscr{V}_{0}\right\}$. Then $\mathscr{W}=\bigcup_{n=1}^{\infty} \mathscr{W}_{n}$ and $\mathscr{Z}=\bigcup_{n=1}^{\infty} \mathscr{Z}_{n}$ are rank 1 collections, and they constitute together a Noetherian rank 2 base for the product of the Michael line with the irrationals. A Noetherian rank 3 base for the product of the Michael line with itself can be constructed in a similar manner.

The Michael line has a $G_{\delta}$-diagonal because the real line has one; but the Michael line is not developable because every paracompact, developable space is metrizable. (One could also show this by using Theorem 4.3.)

EXAMPLE 5.2. The Sorgenfrey line $S$ was introduced in [32] as a paracompact space whose square is not normal. It is obtained by putting the topology on the real line whose base is the set of all upperhalf-open intervals $[a, b)$. The Sorgenfrey line does not have an orthobase. In fact [21] it does not even have a quasi-uniform base.

Let $\mathcal{U}_{n}$ be the set of all intervals $[a, b)$ of length $1 / n$. Then $\mathcal{U}=\cup_{n=1}^{\infty} \mathcal{U}_{n}$ is a Noetherian base for $S$, since any ascending sequence of members of $U$ must have strictly increasing lengths.

We will construct a base of sub-infinite rank for $S$ by using the homeomorphism between $S$ and the subspace of $S$ consisting of the interval 0,1$)$. Let $\left\{q_{i}\right\}$ be a sequence of rational numbers in $(0,1]$ such that for each $n, \bigcup_{i=n}^{\infty}\left(q_{i}-\frac{1}{l}, q_{i}\right)$ is a cover of $[0,1]$. (Note that the lengths of the intervals form a divergent series.) Let $\mathscr{B}_{i}=\left\{\left[x, q_{i}\right) \mid q_{i}-x<\right.$ $\left.{ }_{1}^{1}\right\}$. Let $\mathscr{B}=\cup_{i=1}^{\infty} \mathscr{B}_{i}$. Clearly $\mathscr{B}$ contains a base for $(0,1)$ with the Sorgenfrey topology. We will show that $\mathscr{B}$ is of sub-infinite rank.

Let $\mathscr{A} \subset \mathscr{B}$ be an infinite incomparable collection. This implies $\mathscr{A} \cap \mathscr{B}_{t}$ contains at most one member for each $i$. Let $j(1), j(2), \cdots$ be the enumeration (in numerical order) of the indices $i$ for which $\mathscr{A} \cap$ $\mathscr{B}_{i} \neq \varnothing$. Then $\mathscr{A}=\left\{\left[x_{n}, q_{\jmath_{(n)}}\right)\right\}_{n=1}^{\infty}$. Suppose $p \in \cap \mathscr{A}$. Clearly $p=x_{n}$ for at most one $n$. Then $x_{m}<p$ for $m \neq n$. Choose $m \neq n$ and $k$ such that

$$
1 / k<\min \left\{q_{j(m)}-p, p-x_{m}\right\}
$$


For some $i, j(i)>k$. It is easy to see that $\left[x_{i}, q_{j(i)}\right) \varsubsetneqq\left[x_{m}, q_{j(m)}\right)$, since both contain $p$, and the latter contains all points within a distance $1 / k$ from $p$.

By Theorem 3.5, neither $\mathscr{B}$ nor any other base of sub-infinite rank for $S$ is Noetherian. Indeed, the Sorgenfrey plane $S^{2}$ does not have a base of sub-infinite rank nor even of countable rank: every base for $S^{2}$ is of rank $2^{\mathbf{N}_{0}}=c$.

To see this, let $U$ be a base for $S^{2}$. For each point $(x,-x)$ on the anti-diagonal of $S^{2}$, pick a member $U_{x}$ of $U$ that meets the anti-diagonal only in $(x,-x)$. (Such a $U_{x}$ must exist if $\mathcal{U}$ is to be a base, because the squares $B(x, \delta)=\{(a, b) \mid x \leqq a<x+\delta,-x \leqq b \leqq-x+\delta\}$ form a local base at $(x,-x)$ as $\delta$ ranges over the positive real numbers.) For each $n$, let $\mathscr{U}_{n}=\left\{U_{x} f \mid B(x, 1 / n) \subset U_{x}\right\}$. Since each $U_{x}$ is open, $\cup_{n=1}^{\infty} \mathscr{U}_{n}=$ $\left\{U_{x} \mid x \in S\right\}$. Therefore, there must exist some $U_{n}$ of cardinal c. If we divide up the anti-diagonal into segments of length $\sqrt{2} / n$, disjoint except for their endpoints, there exists at least one that meets $c$ members of $\boldsymbol{U}_{n}$ in a point other than one of the endpoints. Any one of these $c$ members contains the point at a horizontal distance $1 / n$ from the "upper" endpoint of the segment, and each contains a point not to be found in any of the others.

ExAmple 5.3. Let $D_{1}^{*}$ be the discretization of the ordinal $\omega_{1}+1$ obtained by isolating all points except $\omega_{1}$. Clearly, $D_{1}^{*}$ has a Noetherian rank 1 base (although $\omega_{1}+1$ with the linear order topology does not-see Example 5.5). Its product with $\omega_{0}+1$ is known as the Dieudonné plank. Although both factor spaces have Noetherian rank 1 bases, the plank itself does not have a base of any finite rank, because the corner point $\left(\omega_{1}+1, \omega_{0}+1\right)$ does not have a totally ordered base (Lemma 2.4). However, it does have a Noetherian base of sub-infinite rank (Theorem 3.5) and hence is hereditarily metacompact (Theorem 3.3).

The space obtained by deleting the corner point $\left(\omega_{1}+1, \omega_{0}+1\right)$, called the deleted Dieudonné plank, is a nonnormal, metacompact space, [33], [34]. It has a base of rank 2 . Let $\mathscr{B}_{o}$ be the set of all singletons $(\alpha, n)$ with $\alpha$ countable and $n$ finite; let $\mathscr{B}_{n}$ be the set of all vertical segments of the form $B_{n}^{\alpha}=\{(\alpha, \beta) \mid \beta>n\}$ as $\alpha$ ranges over all countable ordinals. Finally, let $\mathscr{B}_{\alpha}$ be the set of all horizontal segments of the form $B_{\alpha}^{n}=\{(\beta, n) \mid \beta \geqq \alpha\}$. Clearly,

$$
\mathscr{B}_{0} \cup\left(\bigcup_{n<\omega_{0}} \mathscr{B}_{n}\right) \cup\left(\bigcup_{\alpha<\omega_{1}} \mathscr{B}_{\alpha}\right)
$$

is a rank 2 base.

Given a regular cardinal $\omega_{\mu}$, one can define $D_{\mu}^{*}$ analogously to $D_{1}^{*}$ as the discretization of $\omega_{\mu}+1$ obtained by isolating all but the final point. Here is an important embeddability principle: if $X$ is a space 
with an ortho-base, and $x$ is a point of $X$ which does not have countable local base, then we can embed some $D_{\mu}^{*}, \omega_{\mu}$ regular and uncountable, as a closed subspace of $X$ whose single nonisolated (in relative topology) point is $x$. The method is a simple transfinite induction argument: for each $\alpha<\omega_{\mu}$, pick a neighborhood $V_{\alpha}$ of $x$ from the ortho-base in such a way that $V_{\beta} \supsetneqq V_{\alpha}$ for all $\beta<\alpha$, and so that $\left\{V_{\alpha}: \alpha<\omega_{\mu}\right\}$ is a local base at $x$. Now for each $\alpha$, pick a point in $V_{\alpha} \backslash V_{\alpha+1}$.

Since $D_{\mu}^{*}$ is neither a $k$-space nor a $q$-space, this argument gives a simple way of establishing Theorem 4.1. A similar use of these spaces is to be found in [27].

EXAMPLE 5.4. In marked contrast to $D_{1}^{*}$, the space $\omega_{1}$ of countable ordinals has no base of sub-infinite rank. (This applies a fortiori to $\omega_{1}+1$.) To prove this, we apply a result of Alexandroff and Urysohn whose proof may be found in [19, Theorem A1.2]. Given an ordinal number $\alpha$ (regarded as the set of all ordinals smaller than $\alpha$ ), a function $f: \alpha \rightarrow \alpha$ is regressive if $f(0)=0$ and for all $\beta>0, f(\beta)<\beta$. The Alexandroff-Urysohn theorem is: If $f: \omega_{1} \rightarrow \omega_{1}$ is regressive, then there exists $\beta<\omega_{1}$ such that $\beta=f(\alpha)$ for uncountably many $\alpha$. In fact, we can find $\beta$ such that $\beta=f(\alpha)$ for uncountably many limit ordinals, because the function that agrees with $f$ on limit ordinals and sends each nonlimit ordinal to its predecessor is also regressive.

Now, let $\mathscr{B}$ be a base for $\omega_{1}$. For each limit ordinal $\alpha<\omega_{1}$, select a member $B_{\alpha}$ of $\mathscr{B}$ whose upper endpoint is $\alpha$. Since $\alpha$ is in the interior of $B_{\alpha}$, there exists $\beta<\alpha$ such that $[\beta, \alpha]=\{\gamma \mid \beta \leqq \gamma \leqq \alpha\}$ is a subset of $B_{\alpha} \quad$ Let $\beta_{1}(\alpha)$ be the least such ordinal. By the Alexandroff-Urysohn theorem, there exists an ordinal $\beta_{1}$ such that $\beta_{1}=\beta_{1}(\alpha)$ for uncountably many $\alpha$. Suppose that $\beta_{n}$ has been selected. For each limit ordinal $\alpha$ greater than $\beta_{n}$, select a member $B_{\alpha}^{n}$ of $\mathscr{B}$ whose upper endpoint is $\alpha$ and whose lower endpoint is greater than $\beta_{n}$. Again there exists $\beta<\alpha$ such that $[\beta, \alpha]$ is a subset of $B_{\alpha}^{n}$. Let $\beta_{n+1}(\alpha)$ be the least such ordinal, and let $\beta_{n+1}$ be such that $\beta_{n+1}=\beta_{n+1}(\alpha)$ for uncountably many $\alpha$.

And so by induction, we define an increasing sequence $\left\{\beta_{n}\right\}$ of ordinals. Let $\beta=\sup \left\{\beta_{n}\right\}$, and pick for each positive $n$ an ordinal $\alpha_{n}$ such that $\beta_{n}=\beta_{n}\left(\alpha_{n}\right)$ in such a way that $\alpha_{n}<\alpha_{m}$ whenever $n<m$, and $\alpha_{1}>\beta$. It is clear that $\beta$ is in the intersection of the sets $B_{\alpha_{n}}^{n}$ and that the sets are incomparable.

On the other hand, the open intervals of $\omega_{1}$ constitute a base of countable rank.

Although $\omega_{1}$ is orthocompact, being a linearly ordered space, it does not have an ortho-base. Indeed, defining $\beta$ as above, we have $\beta \in$ $\bigcap_{n=1}^{\infty} B_{\alpha_{n}}^{n}$, but $\beta$ is not in the interior of the intersection, nor is $\beta$ the only point in the intersection. 
The following proof that $\omega_{1}$ has a Noetherian base is due to $G$. Gruenhage. J. Vaughan and B. Scott have independently arrived at similar proofs. B. Scott has, in addition, given a proof that $\omega_{1}$ does not have a clopen Noetherian base. Also, it is easy to see (using [19, Theorem A 1.2] as above) that $\omega_{1}$ does not have a Noetherian base of intervals.

Let $\mathscr{B}_{0}$ be the set of all isolated points of $\omega_{1}$. For each limit ordinal $\alpha$ that is not a limit of limit ordinals, let $\mathscr{B}_{\alpha}=\left\{\left[\alpha_{p}+n, \alpha\right] \mid n\right.$ is a positive integer, $\alpha_{p}$ is the limit ordinal immediately preceding $\alpha$ (if $\alpha=\omega$, $\left.\left.\alpha_{p}=0\right)\right\}$.

For any limit ordinal $\alpha$, let

$$
\mathscr{F}_{\alpha}=\{F(\alpha, \beta, n) \mid \beta>\alpha, n \in \mathbf{N}\}
$$

where $F(\alpha, \beta, n)$ is defined as follows:

Let $\left\{H_{i}\right\}_{i \in I}$ be an uncountable collection of subsets of $[0, \alpha]$ with the following properties:

(i) $H_{i}$ contains no limit ordinals

(ii) $\alpha$ is the only cluster point of $H_{i}$

(iii) $H_{i} \cap H_{j}$ is at most finite for $i \neq j$.

For each $\beta>\alpha$, choose $H_{i(\beta)}$ so that if $\beta \neq \gamma, i(\beta) \neq i(\gamma)$. Suppose $H_{i(\beta)}=\left\{\delta_{1}, \delta_{2}, \cdots\right\}$. Let

$$
\left.\left.F(\alpha, \beta, n)=\left\{\delta_{n+1}, \delta_{n+2}, \cdots\right\} \cup\right] \alpha, \beta\right] .
$$

Now $\mathscr{B}_{0} \cup\left(\cup_{\alpha<\omega_{1}} \mathscr{B}_{\alpha}\right) \cup\left(\cup_{\alpha<\omega_{1}} \mathscr{F}_{\alpha}\right)$ is a base for $\omega_{1}$. It is Noetherian, because any infinite ascending chain would have to contain an infinite sequence

$$
F\left(\alpha_{1}, \beta_{1}, n_{1}\right) \subset F\left(\alpha_{2}, \beta_{2}, n_{2}\right) \subset \cdots
$$

Then $\alpha_{1} \geqq \alpha_{2} \geqq \cdots$ so eventually $\alpha_{n}=\alpha_{0}$ for some $\alpha_{0}$ and all $n$ greater than some integer $N$. By property (iii) we must have all $\beta_{n}$ equal for all $n \geqq N$. Thus $n_{N}>n_{N+1}>\cdots$ and so the sequence must be finite.

EXAmPle 5.5. Our final example is an ordinal space with no Noetherian base, due to J. Vaughan.

Let $\kappa$ be a strong limit cardinal (i.e. for every cardinal $\tau<\kappa, 2^{\tau}<\kappa$ ) of uncountable confinality. (For example, under the Generalized Continuum Hypothesis, $\boldsymbol{N}_{\omega_{1}}$ is the first such cardinal.) For all $\alpha<\kappa$, let $I_{\alpha}$ be an interval of the form $\left(x_{\alpha}, \alpha\right]$ with $x_{\alpha}<\alpha$.

Claim. There exists an infinite $\beta<\kappa$, and $A \subset \kappa$, such that $|A|>2^{\beta}$ 
and, for all $\alpha \in A, I_{\alpha} \cap[0, \beta] \neq \varnothing$. (The proof of this claim may be found in [19, Theorem A1.3].)

Now, if $\mathscr{B}$ is a base for $\kappa$, then there exists for each $\alpha<\kappa$ a member $B_{\alpha}$ of $\mathscr{B}$ of the form $T_{\alpha} \cup I_{\alpha}$ where $y<x_{\alpha}$ for all $y \in T_{\alpha}$. Let $\beta, A$ be as in the above claim. Select a countable subset of $A: \alpha_{1}<\alpha_{2}<\cdots$ such that $B_{\alpha_{i}} \cap[0, \beta]=B_{\alpha_{l}} \cap[0, \beta]$ for all $i, j$. (This is possible since $|A|>$ $2 \beta$.) It is then clear that $B_{\alpha_{i}} \varsubsetneqq B_{\alpha_{i+1}}$ for all $i$. (Indeed, $B_{\alpha_{i}}$ contains all ordinals between $\beta$ and $\alpha_{i}$, but no ordinal greater than $\alpha_{i}$.) It follows that $\mathscr{B}$ is not Noetherian.

6. Open questions. In light of Theorems 3.5 and 3.3, it is of interest to inquire:

Problem 6.1. Does every space with base of finite rank have a Noetherian base of finite [equivalently, sub-infinite] rank?

A space with a rank 1 base has a Noetherian rank 1 base [25], but Problem 6.1 is open for spaces with bases of rank 2. The explanation of the "equivalently" in Problem 6.1 is that the space with a Noetherian base $\mathscr{B}$ of sub-infinite rank and a base $\mathcal{V}$ of finite rank $n$ has a Noetherian base of rank $n$. This can be shown by using Theorem 3.8: assume $\mathscr{V}$ is upper-chain-complete, and for each $B \in \mathscr{B}$ and each $p \in B$, take the set of maximal members of $\mathscr{V}$ contained in $B$ and containing $p$. The set of all such members of $\mathscr{V}$ is clearly a base of rank $n$, and is Noetherian because $\mathscr{B}$ is Noetherian and of sub-infinite rank [Noetherian alone is not enough].

Problem 6.1 is important because an affirmative answer would greatly shorten the present proofs that every Hausdorff or separable regular space with a base of finite rank is metrizable; that every space with a base of finite rank is metacompact; that every hereditarily Lindelöf space with a base of finite rank has a point-countable base [14]. It would also provide an affirmative answer to the following question.

Problem 6.2. Let $X$ be a space which is a finite product of spaces with bases of finite rank. Is $X$ metacompact? hereditarily metacompact?

Problem 6.3. Is every space with a base of sub-infinite rank metacompact?

This problem is a natural outgrowth of several results, for example Corollary 2.8 or Theorem 3.3 or the result of P. Nyikos [14] that every space with a base of rank $\boldsymbol{N}_{0}--$ is metacompact. 
The following is equivalent to Problem 6.3: is every space with a base of sub-infinite rank $\theta$-refinable? Indeed, metacompactness is equivalent to $\theta$-refinability for spaces with bases of sub-infinite (small) rank, because these are all "pointwise collectionwise normal" (almost discretely expandable) and J. R. Boone [5] has shown that $\theta$-refinability and metacompactness are equivalent in such spaces.

An affirmative answer to Problem 6.2 would yield the following strengthening of Theorem 2.9: A space is metacompact and developable if, and only if, it has a base of countable order and a base of sub-infinite rank.

Problem 6.4. Is every space with a base of countable rank orthocompact?

As remarked above, $\omega_{1}$ is a linearly ordered space with a base of countable rank that is not metacompact.

Problem 6.5. Is every countably compact space with a base of sub-infinite rank compact?*

A similar, though probably unrelated problem is:

Problem 6.6. Is every countably compact space with an ortho-base compact ?*

Exercise 5I of [12] gives a pseudocompact space with an ortho-base that is not compact-but it is not countably compact, either.

The following problem from [15] remains unanswered:

Problem 6.7. Is every collectionwise normal space with an orthobase paracompact?

Even the following problem remains unsolved. Of course, any orthocompact nonmetrizable normal Moore space would provide a counterexample. (That is hardly the only place to look!)

Problem 6.8. Is there a model of set theory in which every normal space with an ortho-base is paracompact?

As shown by Theorem 4.4, the following implications hold for a space with an ortho-base:

$$
\sigma^{*} \text {-space } \Rightarrow \text { quasi-metric space } \Rightarrow \gamma \text {-space }
$$

\footnotetext{
* Added in proof. G. Gruenhage has shown that both 6.5 and 6.6 have affirmative answers.
} 


\section{Problem 6.9. Which of the above implications is reversible for} every space with an ortho-base?

G. Gruenhage has shown that if the space is paracompact, both implications reverse [13].

\section{REFERENCES}

1. P. S. Alexandroff, On the metrization of topological spaces, Bull. Acad. Polon. Math. Ser., 8 (1960), 135-140 (Russian).

2. A. V. Arhangel'skii and V. V. Filippov, Spaces with bases of finite rank, Math USSR Sbornik, 16 (1972), 147-158.

3. R. H. Bing, Metrization of topological spaces, Canad. J. Math., 3 (1951), 175-186.

4. G. Birkhoff, Lattice Theory, 3rd ed., Providence, Amer. Math. Soc., 1967.

5. J. R. Boone, A characterization of metacompactness in the class of $\theta$-refinable spaces, General Topology Appl., 3 (1973), 253-264.

6. M. M. Čoban, Spaces with bases of rank one, Bull. Akad. RSS Moldovenest, 3 (1973), 12-19, 91 (Russian).

7. R. P. Dilworth, A decomposition theorem for partially ordered sets, Annals of Math., 51 (1950), 161-16

8. R. Engelking, Outline of General Topology, Amsterdam, North Holland, 1968.

9. P. Fletcher and W. F. Lindgren, Orthocompactness and strong Čech completeness in Moore spaces, Duke Math J., 39 (1972), 753-766.

10. L Locally quasi-uniform spaces with countable bases, Duke. Math. J., 41 (1974), 231-240.

11. _- Transitive quasi-uniformities, J. Math. Anal. Appl., 39 (1972), 397-405.

12. L. Gillman and M. Jerison, Rings of Continuous Functions, Princeton, Van Nostrand, 1960.

13. G. Gruenhage, A note on quasi-metrizability (preprint).

14. G. Gruenhage and P. Nyikos, Spaces with bases of countable rank, (to appear).

15. R. W. Heath, Arc-wise connectedness in semi-metric spaces, Pacific J. Math., 12 (1962), 1301-1319.

16. R. E. Hodel, Spaces defined by sequences of open covers which guarantee that certain sequences have cluster points, Duke Math. J., 39 (1972), 253-263.

17. - Moore spaces and $w \Delta$-spaces, Pacific J. Math., 38 (1971), 641-652.

18. — Metrizability of topological spaces, Pacific J. Math., 55 (1974), 441-459.

19. I. Juhász, Cardinal Functions in Topology, Mathematical Centre Tracts No. 34, Math. Centrum, Amsterdam, 1971.

20. Ya. A. Kofner, On $\Delta$-metrizable spaces, Math. Notes Acad Sci. USSR, 13 (1973), 168-174.

21. D. J. Lutzer, On quasi-uniform bases, Proceedings of the University of Oklahoma Topology Conference, Norman, Oklahoma, 1972.

22. E. Michael, The product of a normal space and a metric space need not be normal, Bull. Amer. Math. Soc., 69 (1963), 375-376.

23. J. Nagata, On dimension and metrization, General Topology and its Relations to Modern Analysis and Algebra, New York, Academic Press, 1962.

24. - Modern General Topology, Amsterdam, North Holland, 1968.

25. P. Nyikos, Some surprising base properties in topology, Studies in Topology, New York, Academic Press, 1975.

26. P. Nyikos and H.-C. Reichel, On the structure of zero-dimensional spaces, Indag. Math., 37 (1975), 120-136.

27. - On uniform spaces with linearly ordered bases II ( $\omega_{\mu}$-metric spaces), Fund. Math., 93 (1977), 1-10.

28. - Non-archimedean spaces and some generalizations, (in preparation). 
29. H. Ribeiro, Sur les espaces a metrique faibile, Portugaliae Math., 4 (1943), 21-40.

30. B. Scott, Toward a product theory for orthocompactness, Studies in Topology, New York, Academic Press, 1975.

31. F. Siwiec and J. Nagata, A note on nets and metrization, Proc. Japan Acad., 44 (1968), 623-627.

32. R. H. Sorgenfrey, On the topological product of paracompact spaces, Bull. Amer. Math. Soc., 53 (1947), 631-632.

33. L. A. Steen and J. A. Seebach, Counterexamples in topology, New York, Holt, Rinehart, and Winston, 1970.

34. J. Vaughan, Linearly ordered collections and paracompactedness, Amer. Math. Soc. Proceedings, 24 (1970), 186-192.

35. H. H. Wicke and J. M. Worrell, Characterizations of developable topological spaces, Canad. J. Math., 17 (1965), 820-830.

36. H. H. Wicke and J. M. Worrell, Topological completeness of first countable Hausdorf spaces I, Fund. Math., 75 (1972), 209-222.

Received July 1, 1975.

Slippery Rock State College

AND

UNIVERSITY OF ILLINOIS, URBANA

Current address of: P. J. Nyikos

Auburn University

Auburn, AL 36830 


\section{PACIFIC JOURNAL OF MATHEMATICS}

\section{EDITORS}

RICHARI) ARENS (Managing Editor)

University of California

Los Angeles, CA 90024

R. A. Beaumiont

University of Washington

Seattle, WA 98105

\section{J. DugunduI}

Department of Mathematics University of Southern California Los Angeles, CA 90007

D. Gilbarg and J. Milgram Stanford University

Stanford, CA 94305

\section{ASSOCIATE EDITORS}

E. F. BECKENBACH
B. H. NEUMANN

F. Wolf

K. YoshidA

\section{SUPPORTING INSTITUTIONS}

UNIVERSITY OF BRITISH COLUMBIA CALIFORNIA INSTITUTE OF TECHNOLOGY

UNIVERSITY OF CALIFORNIA

MONTANA STATE UNIVERSITY

UNIVERSITY OF NEVADA

NEW MEXICO STATE UNIVERSITY

OREGON STATE UNIVERSITY

UNIVERSITY OF OREGON

OSAKA UNIVERSITY

\author{
UNIVERSITY OF SOUTHERN CALIFORNIA \\ STANFORD UNIVERSITY \\ UNIVERSITY OF HAWAII \\ UNIVERSITY OF TOKYO \\ UNIVERSITY OF UTAH \\ WASHINGTON STATE UNIVERSITY \\ UNIVERSITY OF WASHINGTON \\ AMERICAN MATHEMATICAL SOCIETY
}

The Supporting Institutions listed above contribute to the cost of publication of this Journal, but they are not owners or publishers and have no responsibility for its contents or policies.

Mathematical papers intended for publication in the Pacific Journal of Mathematics should be in typed form or offset-reproduced (not dittoed), double spaced with large margins. Underline Greek letters in red, German in green, and script in blue. The first $p: 1<$ graph or two must be capable of being used separately as a synopsis of the entire paper. Items of the biblography should not be cited there unless absolutely necessary, in which case they must he identified by author and Journal, rather than by item number. Manuscripts, in duplicate, may be sent to any one of the four editors. Please classify according to the scheme of Math. Reviews, Index to Vol. 39. All other communications should be addressed to the managing editor, or Elaine Barth, University of California, Los Angeles, California, 90024.

100 reprints are provided free for each article, only if page charges have been substantially paid. Additional copies may be obtained at cost in multiples of 50 .

The Pacific Journal of Mathematics is issued monthly as of January 1966. Regular subscription rate: $\$ 72.00$ a year (6 Vols., 12 issues). Special rate: $\$ 36.00$ a year to individual members of supporting institutions.

Subscriptions, orders for back numbers, and changes of address should be sent to Pacific Journal of Mathematics, 103 Highland Boulevard, Berkeley, California, 94708.

PUBLISHED BY PACIFIC JOURNAL OF MATHEMATICS, A NON-PROFIT CORPORATION Printed at Jerusalem Academic Press, POB 2390, Jerusalem, Israel.

\section{Copyright (C) 1976 Pacific Journal of Mathematics} All Rights Reserved 


\section{Pacific Journal of Mathematics}

\section{Vol. 66, No. 2 December, 1976}

Gerald A. Beer, Tax structures whose progressivity is inflation neutral..... 305

William M. Cornette, A generalization of the unit interval............. 313

David E. Evans, Unbounded completely positive linear maps on

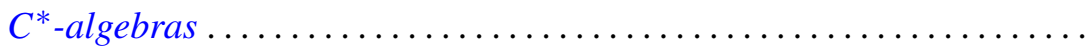

Hector O. Fattorini, Some remarks on convolution equations for

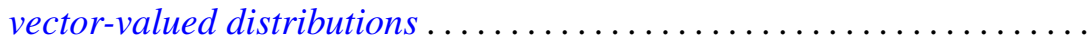

Amassa Courtney Fauntleroy, Automorphism groups of unipotent groups of

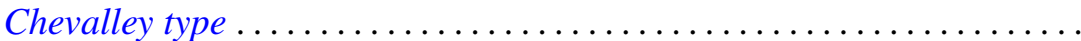

Christian C. Fenske and Heinz-Otto Peitgen, On fixed points of zero index in

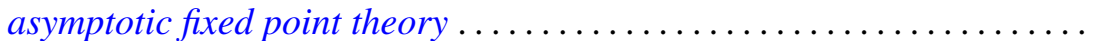

Atsushi Inoue, On a class of unbounded operator algebras. II ............

Herbert Meyer Kamowitz, The spectra of endomorphisms of algebras of

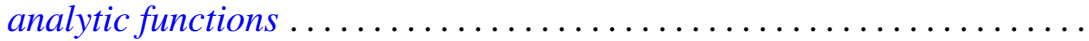

Jimmie Don Lawson, Embeddings of compact convex sets and locally compact cones ....................................

William Lindgren and Peter Joseph Nyikos, Spaces with bases satisfying certain order and intersection properties .....................

Emily Mann Peck, Lattice projections on continuous function spaces ...... 477

Morris Marden and Peter A. McCoy, Level sets of polynomials in $n$ real variables...

Francis Joseph Narcowich, An imbedding theorem for indeterminate Hermitian moment sequences......................

John Dacey O'Neill, Rings whose additive subgroups are subrings ...

Chull Park and David Lee Skoug, Wiener integrals over the sets bounded by sectionally continuous barriers .....................

Vladimir Scheffer, Partial regularity of solutions to the Navier-Stokes equations.

Eugene Spiegel and Allan Trojan, On semi-simple group algebras. II

Katsuo Takano, On Cameron and Storvick's operator valued function space integral 